\title{
Neuropsychological \& Cognitive Sequelae in COVID-19 Patients
}

\author{
Yunier Broche-Pérez MS PhD and Claudia M. Medina-Navarro MS
}

A neuroinvasive respiratory coronavirus, SARS-CoV-2 causes a variety of neurological responses in patients. These include dizziness, headache, myalgias, hypogeusia, hyposmia, polyneuropathy, myositis, cerebrovascular disease, encephalitis and encephalopathy. Such susceptibility of the central nervous system (CNS) to the virus has spurred interest in neuropsychological research in COVID-19 patients and convalescents.

Earlier health crises provide evidence confirming the adverse effects of respiratory diseases on cognitive function. For example, some $15 \%$ of patients infected with Severe Acute Respiratory Syndrome (SARS) or Middle East Respiratory Syndrome (MERS) showed deficits in cognitive functions like memory and attention.[1]

Preliminary results from the current pandemic suggest that SARSCoV-2 infection also negatively impacts neuropsychological function in COVID-19 convalescents.[2] Those processes shown to be adversely affected include sustained attention, memory, working memory, cognitive flexibility and verbal fluency.

People with neurodegenerative diseases are among the most vulnerable for developing cognitive disorders as a result of COVID-19 infection. These include patients with Huntington's disease, amyotrophic lateral sclerosis (ALS), Alzheimer's disease, multiple sclerosis and Parkinson's disease, who will require meticulous cognitive follow-up during COVID-19 convalescence. [3] Furthermore, early evidence suggests that patients who present with metabolic disturbances and test positive for COVID-19 could be at higher risk for cognitive decline and dementia later in life.[4]

\begin{tabular}{|l|} 
Research is still lacking \\
on how COVID-19 \\
infection affects \\
cognitive functioning
\end{tabular}

Despite these early findings, research is still lacking on how COVID-19 infection affects cognitive functioning. In our opinion, various factors contribute to the dearth of neuropsychological studies among convalescent patients. First and foremost, studies examining SARS-CoV-2 sequelae focus primarily on clinical variables, particularly respiratory and neurological onesunderstandable given the gravity of the pandemic declared in 2020. And while the scope of COVID-19 research is expanding, much is still unknown about the disease's medium and long-term complications. Second, confinement measures designed to control transmission have multiplied the pressure on and demand for psychology and neuropsychology services, while making it more difficult to provide face-to-face neuropsychological diagnosis and rehabilitation. Finally, the multiplicity of variables related to COVID-19 that are also linked to cognitive function is another challenge for research. These variables include but are not limited to: disease progression; treatment protocols; patients' emotional response following a positive diagnosis and through convalescence; and physical and psychological complications.

In our work as neuropsychological researchers and clinicians, we measure cognitive function, but also examine variablesdirect and indirect-and their effect on higher nervous activity. Increasingly, convalescent COVID-19 patients who access our services complain of deficits in memory, sustained attention and processing speed, among other problems. These patients worry about how these cognitive impairments affect their daily lives and come to us in search of treatment to help them manage these difficulties.

In our daily practice, we have seen that cases can vary widely, from patients with only mild symptoms to others who required mechanical ventilation; some who suffer from severe depression, while fatigue predominates in others; and in several instances, patients stated they have not experienced cognitive decline but clinical evaluation showed otherwise, revealing decreased mental performance in one or more cognitive domains. Given the differences in clinical history and disease progression, each convalescent patient will need meticulous follow-up and individualized neuropsychological treatment.

We cannot overstate the importance of collaboration between neuropsychology researchers and clinicians when attending COVID-19 patients. This type of collaboration is imperative and led us to develop criteria for transversal research taking into account the following variables:

1. Neurological manifestations in COVID-19 patients from diagnosis to hospital discharge, documenting duration and if they continue during convalescence, paying particular attention to neurological changes related to the CNS and peripheral nervous system (PNS);

2. Links between illness severity and level of cognitive impairment-preliminary research indicates that severe COVID-19 patients suffer more serious neurological symptoms and may experience greater neuropsychological changes as a result of infection; [5] and

3. Potential effects of COVID-19 treatment on neuropsychological functioning.

The link between emotional state and cognitive function is another, well-documented, variable that must be considered in future cross-sectional research-specifically manifestations of anxiety and depression. Special attention should be paid to older adults recovering from COVID-19 since depression is linked to significant cognitive decline and is a risk factor for dementia.

Longitudinal studies monitoring cognitive impairment in the short, medium and long term should also be prioritized, bearing in mind that neuropsychological dysfunction can last months or even years after recovery from COVID-19. Documenting the severity of cognitive deficits over time will facilitate design and implementation of appropriate neuropsychological interventions for these patients.

We also recommend conducting baseline studies that examine a minimum of five cognitive domains: attention, memory, executive functions, language and visuospatial functions. These should be monitored and measured using reliable, valid instruments that control for the learning effect. Patients should be evaluated every 3 to 6 months for a minimum of 18 months. 
Finally, it's important that future research explore the impact cognitive decline has on daily life over time. Measuring how cognitive and non-cognitive variables affect quality of life, in realworld settings, can help provide ecological validity to these baseline studies. As a tool, we recommend the Post-COVID-19 Functional Status Scale (PCFS) to evaluate how dyspnea, fatigue, memory loss, anxiety, depression and other problems limit daily activities. [6] By measuring and understanding COVID-19 infection's impact on cognitive function, researchers can evaluate the effectiveness of pharmacological (primarily psychoactive drugs) and non-pharmacological (psychotherapy, neuropsychological rehabilitation) interventions for these patients.

In our opinion, thorough, comprehensive control and follow-up incorporating these variables is fundamental for facing the biggest challenge to cognitive health and well-being facing COVID-19 convalescents: neuropsychological rehabilitation. -1 .

\section{REFERENCES}

1. Rabinovitz B, Jaywant A, Fridman CB. Neuropsychological functioning in severe acute respiratory disorders caused by the coronavirus: implications for the current COVID-19 pandemic. Clin Neuropsychol. 2020 Oct-Nov;34(78):1453-79.

2. Ferrucci R, Dini M, Gropo E, Rosci C, Reitano MR, Bai F, et al. Long-lasting cognitive abnormalities after COVID-19. Brain Sci. 2021 Feb 13;11(2):235

3. Serrano-Castro PJ, Estivill-Torrús G, Cabezudo-García P, Reyes-Bueno JA Ciano Petersen N, Aguilar-Castillo MJ, et al. Impact of SARS-CoV-2 infection on neurodegenerative and neuropsychiatric diseases: a delayed pandemic? Neurología. 2020 May;35(4):245-51. English, Spanish.
4. Miners S, Kehoe PG, Love S. Cognitive impact of COVID-19: looking beyond the short term. Alzheimer's Res Ther. 2020 Dec 30;12(1):170.

5. Beaud V, Crottaz-Herbette S, Dunet V, Vaucher J, Bernard-Valnet R, Du Pasquier R, et al. Pattern of cognitive deficits in severe COVID-19. J Neurol Neurosurg Psychiatry. 2020 Nov 20:325173. DOI: 10.1136/jnnp-2020-325173

6. Klok FA, Boon GJAM, Barco S, Endres M, Geelhoed JJM, Knauss S, et al. The Post-COVID-19 Functional Status (PCFS) Scale: a tool to measure functional status over time after COVID-19. Eur Resp J. 2020 Jul 2;56(1):2001494. DOI: $10.1183 / 13993003.01494-2020$

\section{THE AUTHORS}

Yunier Broche-Pérez (Corresponding author: yunierbroche@gmail.com), professor of psychology with a master's degree in medical psychology and a PhD in psychological sciences. Psychology department, Las Villas Central University. Coordinator of the Cuban Cognitive Health Initiative (CogniciON). Member, National Psychology Sciences Tribunal. https:// orcid.org/0000-0002-3450-7374

Claudia Madeleine Medina-Navarro, neuropsychologist with master's degrees in neuropsychology and pediatric neuropsychology, doctoral candidate in psychology sciences. Coordinator, Memory and Cognition Center and member, Evaluation Committee Surgery for Parkinson's disease deep brain stimulation, Hospital Ángeles, Puebla, México. Member, Board of Directors, Colegio Angelopolitano de Neurociencias, Puebla, Mexico. https://orcid.org/0000-0001-7284-8455

Submitted: March 12, 2021

Approved for publication: April 2, 2021

Disclosures: None 